\title{
A Novel Canny Mean Restoration Algorithm for Correspondence and Motion Tracking in Dynamic Image Sequence Analysis
}

\author{
G. S. Yogananda \\ Dept of Electronics and \\ Communication Engg. \\ Kalpataru Institute Of Technology, \\ Tiptur
}

\author{
Y. P. Gowramma \\ Dept.Of Computer \\ Science \& Engg. \\ Kalpataru Institute Of Technology, \\ Tiptur
}

\begin{abstract}
This paper proposes a novel computationally efficient canny mean restoration algorithm for correspondence to identify the matching of the similar features between the reference image frames to the set of search image frames in the dynamic image sequence analysis for tracking motion object in the sequence. This restoration and correspondence has three major steps such as segmentation, feature extraction for restoration, and matching. This paper proposes block-based segmentation in which the reference image frame which contains the object to be tracked can be blocked as square window of size $\mathrm{m} * \mathrm{~m}$ which covers the object. The next step is the feature extraction for restoration. Here we have considered the block based features in which we calculate the canny edge mean of the region of interest. These features are invariant motion blur and noise of motion deblurring, denioseing and it reduces the dimensionality and finally for matching we made use of the minimum absolute similarity distance measure for these features of the blocks. The searching space is restricted to [$15,+15]$ pixels in horizontal ,vertical and diagonal directions in the search image frames. The performance of the algorithm is presented for without preprocessed for slow moving objects of various sequences.
\end{abstract}

\section{Keywords}

Canny Mean, Restoration, Correspondence, Motion tracking.

\section{INTRODUCTION}

Image is a $2 \mathrm{D}$ function $\mathrm{f}(\mathrm{x}, \mathrm{y})$ where $\mathrm{x} \& \mathrm{y}$ are planar coordinates. Where $\mathrm{x}, \mathrm{y} \& \mathrm{f}$ are discrete image is called as digital image. A digital image consists of fixed number of elements having particular value \& location. These elements are called as Pixels or picture elements. Image Restoration is one of the very important techniques in image processing to improve appearance of image. This is done using various techniques like Adaptive filtering, Median filtering etc.

Blurring is one such phenomenon which requires image restoration. Blurring is a kind of Bandwidth reduction of image because of imperfect image formation process. This may result due to relative motion between original image and camera. The blurring process is generalized as convolution of the original image and point spread function(PSF) with additive noise.

$\mathrm{g}(\mathrm{x}, \mathrm{y})=\mathrm{f}(\mathrm{x}, \mathrm{y}) * \mathrm{~g}(\mathrm{x}, \mathrm{y})+\eta$

where $\mathrm{g}(\mathrm{x}, \mathrm{y})$ is degraded image, $\mathrm{f}(\mathrm{x}, \mathrm{y})$ is original image $\mathrm{g}(\mathrm{x}, \mathrm{y})$ is PSF and $\eta$ is additive noise.

There are many reasons for blurring such as Atmospheric Turbulence, Bad focus, motion between camera and original image. The degrations may be due to sensor motion, blur due to cameras focus, relative object camera motion etc. An image can be degraded or corrupted due to various reasons like improper opening of the shutter, atmospheric disturbances, misfocusing etc. Image noise is the random variation of brightness or color information in the image produced by the sensor and circuitry of the scanner and digital camera. Image noise is considered as an undesirable byproduct of image capture. Some types of image noise are Gaussian noise, salt and pepper noise, speckle noise, Brownian noise and many more.

Deconvolution is a long standing problem in many of the application in signal and image processing such as motion pictures (dynamic images), biomedical imaging, astronomy, remote sensing(satellite image processing), super resolution images, forensic images, biometric images and many more. The list is no means exhaustive.

There are many different filter available for deblurring such as mean filter, median filter and adaptive filter which restores images corrupted with noise,

In the motion tracking and correspondence in dynamic scene analysis, image sequence in degraded or blurred due to different types of sequences such as moving object and static sensors, static object \& moving sensors, both object \& moving sensors.

Tracking or surveillance are important for the security, detecting unlikely events, crime detection in the places like hospitals, schools, corridors, offices, banks, aero drums and many more places. All the sequences undergoes motion, motion undergoes degraded due to many reasons and uncontrolled light sources.

In this research paper we have proposed the canny mean restoration feature correspondence for dynamic scene analysis. Restoration of degraded image sequence for correspondence of dynamic scene analysis is one of the recent hot topics of research in the current trend.

Our research proposes the novel method of restoration for moving image sequence for outdoor image sequence, indoor corridor sequence, face tracking pose change sequence, non rigid body sequence.

\section{RELATED WORK}

Hidetomo Sakanio and Xiqum Lu [25] in 2009 reported a study of spatio temporal derivation sensors to extract both the static edges and the dynamic edges from an input video clip and the static edges refers to those caused by spatial intensity variation while the dynamic edges refers the motion traces of both rigid and non-rigid moving objects in the scenes. 
To establish the correspondence, keeping the motion coherence between consecutive frames is very important to track the traces of moving objects or region. In this work they considered canny edge of the image $\mathrm{I}(\mathrm{x}, \mathrm{y}, \mathrm{t})$ and the eigen vector corresponding to the smallest eigen values of canny gradient $\mathrm{G}$ which correspondence to the orientation of the spatio temporal variations of canny dynamic edges are considered as the features.

In the section to follow we discuss about the segmentation based on canny edge detection.

\section{THEORETICAL BACKGROUND}

The segmentation is [41][42] based on three principal concepts: (a) detection of discontinuities (b) thresholding and (c)region processing. Each of these approaches was found to have advantages (for example, speed in the case of global thresholding ) and disadvantages (for example, the need for post processing, such as edge linking, in methods based on detecting discontinuities in gray levels). Segmentation by watersheds embodies many of the concepts of the other three approaches and as such, often produces more stable segmentation results, including continuous segmentation boundaries. This approach also provides a simple framework for incorporating knowledge based constraints in the segmentation process.

\subsection{Canny Edge Detector}

This is based on first derivative coupled with noise cleaning [41],[42]. Therefore, the noise smoothing improves the accuracy of edge detection while adding uncertainty in localizing the edge. Canny edge detector tries to achieve an optimal trade-off between the first derivative and second derivative by approximating the first derivative of the Gaussian. Notice the similarity between the shape of the first derivative of Gaussian and the second derivative of the intensity corresponding to a ramp edge. The derivative of the bell shape of the Gaussian function approximates second derivative or zero crossing operator. Canny has considered the following criteria for localizing edges:

- Good detection: There should be a low probability of failing to detect a real edge point and equivalently, low probability of falsely marking non-edge points. That is to maximize the signal to noise ratio(SNR).

- Good localization: The point marked by the operator as edge points should be as close as possible to the real edge point. That is minimizing the variance $\sigma^{2}$ of the zero crossing position.

- One response to one edge: The detector should not generate multiple outputs in response to a single edge. That is, there should be low probability of number of peaks to a given edge response.

Given the good detection, localization and uni-response to a true edge, the algorithm steps for Canny edge detection are as follows:

Step 1.Convolve the image $\mathrm{g}(\mathrm{r}, \mathrm{c})$ with a Gaussian function (select appropriate $\sigma$ ) to get smooth image g1(r,c), i.e.

$\mathrm{g} 1(\mathrm{r}, \mathrm{c})=\mathrm{g}(\mathrm{r}, \mathrm{c}) * \mathrm{G}(\mathrm{r}, \mathrm{c} ; \sigma)$

Step 2. Apply first difference gradient operator to compute edge strength.

$\mathrm{d} 1=1 / 2\{\mathrm{~g} 1(\mathrm{r}, \mathrm{c})-\mathrm{g} 1(\mathrm{r}, \mathrm{c}-1)+\mathrm{g} 1(\mathrm{r}-1, \mathrm{c})-\mathrm{g} 1(\mathrm{r}-1, \mathrm{c}-1)\}$ $\mathrm{d} 2=1 / 2\{\mathrm{~g} 1(\mathrm{r}, \mathrm{c})-\mathrm{g} 1(\mathrm{r}-1, \mathrm{c})+\mathrm{g} 1(\mathrm{r}, \mathrm{c}-1)-\mathrm{g} 1(\mathrm{r}-1, \mathrm{c}-1)\}$ then edge magnitude and direction are obtained as before.

Step 3. Apply non-maximal suppression to the gradient magnitude. This is achieved by suppressing the edge magnitude not in the direction of the gradient. In fact, in Canny's approach, the edge direction is reduced to any one of the four directions. To perform this task for a given point, its gradient is compared with that of points of its $3 \times 3$ neighborhood. If the candidate magnitude is greater than that of neighborhood, the edge strength is maintained, else it is discarded.

Step 4. Apply threshold to the non-maxima suppressed image. Similar to any other edge - detection process, the edge magnitude below a certain value is discarded. However, the canny's approach employs a clever double thresholding commonly referred to as hysteresis. In this process, two thresholds, upper and lower thresholds are set by the user so that for a given edgel chain if the magnitude of any one edge of the chain is greater than the upper thresholds, all edges above the lower thresholds are selected as edge points. Canny has not provided any basis for selecting upper and lower thresholds and similar to many such applications, selection of the thresholds is application dependent.

Based on the above concepts the proposed methodology brings in efficient feature based correspondence solution.

\section{PROPOSED METHODOLOGY}

In this section we have discussed the proposed methodology in detail. The proposed methodology has three steps such as extracting the region of interest from the reference image, feature extraction and restoration, correspondence or matching.

\subsection{Extracting Region of Interest}

In this step the region of interest or the object of interest is manually initialized using the minimum bounded rectangle, which just touches the object boundary in all four sides of the rectangle in the reference image frame.

\subsection{Restoration and Feature extraction}

As described in the theoretical background of the methodology, the features are extracted from the reference image. In the first stage canny edge of the region of interest is identified. Consequently the watershed edge of the region of interest is identified. Finally mean and variance of both the edges are evaluated separately. It is illustrated in the Figure 1 .

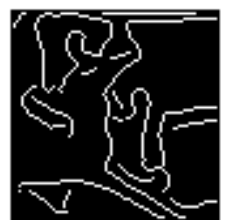

Fig 1. Canny of Region of Interest of the animal sequence

Features considered in this part of the research work are:

Canny edge (Region of interest)

The function Canny edge (Region of interest) finds the only canny edge pixels of the region of interest required the correspondence.

Mean (Canny region pixels intensity)

The functions Mean (Canny region pixels intensity) finds the mean of the canny edge intensities. 
As described in the literature the canny eigen values, canny smallest eigen value vectors and three largest eigen values are used as feature, which are highly time consuming because it requires to solve the higher order polynomials. The order of the polynomial is equal to the size of the region of interest.

Further, the algorithm evaluates the features of the search image or target image. It evaluates the features of searching region. Here the searching region allowed is $[15,+15]$ pixels horizontal , vertical and diagonal regions. In this first few consecutive frames are considered for correspondence. Here the searching space allowed is 15 pixels in all the directions.

\subsection{Correspondence and Motion Tracking}

The matching establishes the correspondence between features of the region of interest in the reference image and same region in search or target image frames such that they both are images of the same physical point. In matching stage minimum absolute difference criterion is used to map most similar regions in the search image frames.

If (minimum absolute (f1(Canny mean)) - $\mathrm{f} 2, \mathrm{f3}$,f4 Canny mean ....) $<$ Threshold

Then

Move the correspondence window to most matching region.

Else

No correspondence

Where f1 refers to feature of reference image region of interest and $f 2, f 3, \ldots$. refers to features of the search region

Figure 2 shows the steps and flow diagram of the canny edge mean based method

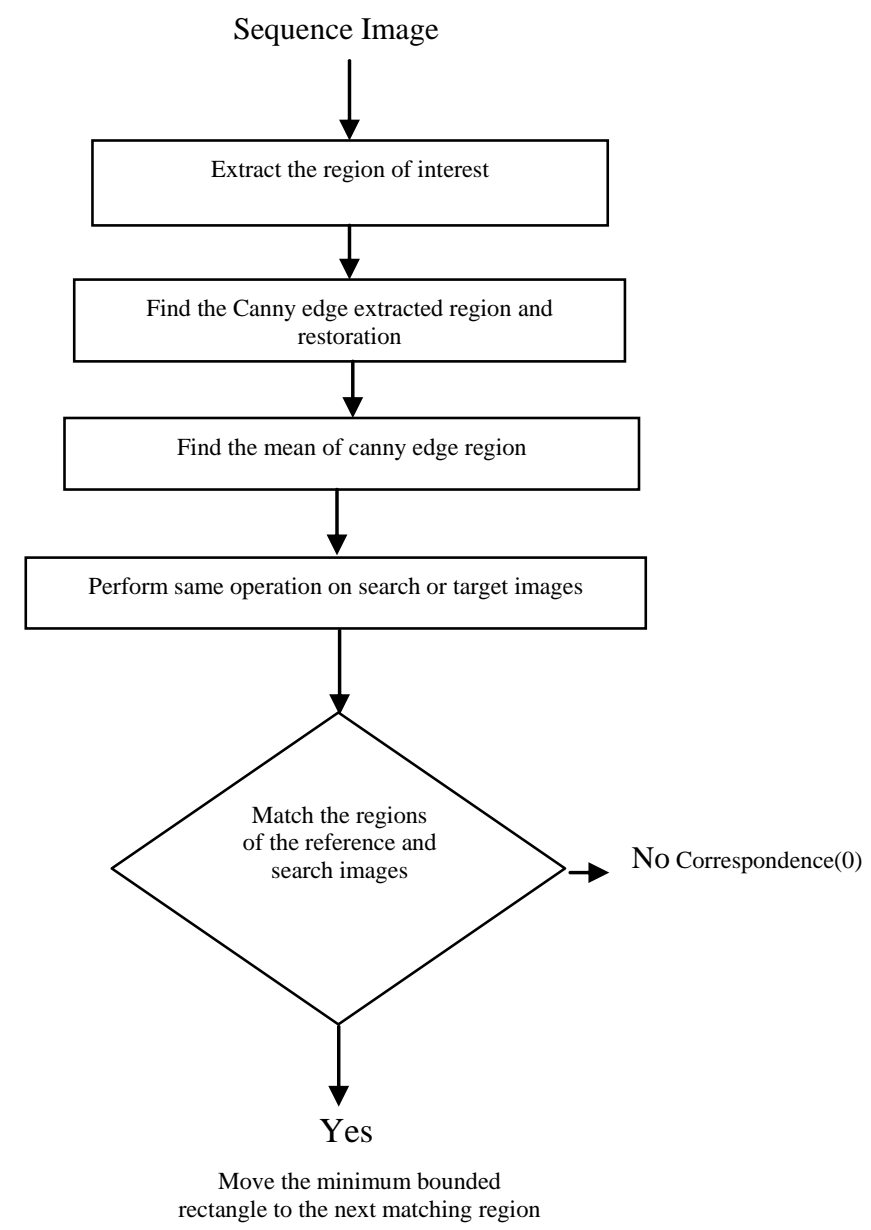

Fig 2. Canny Mean Correspondence system

The algorithm 1 presents the overall methodology

The input and output of the proposed methodology is as follows

Input: Image sequence required correspondence to find the region of interest

Output: The minimum bounded rectangle represents the region of interest in the consecutive image frames.

Algorithm 1 Canny Edge mean Correspondence

Step 1. Read the dynamic image sequence frame.

Step 2. Identify the reference image.

Step 3. Cerate the minimum boundary rectangle box for the region of interest or the object of interest.(need not to be single object).

Step 4. Find the canny of the region of interest.This itself considered as restoration. Find the mean of the canny contours.

Step5. Compare the edge mean of the reference image and the search image.

Step 6. Move the window to the matching region else No correspondence. 
Step 7. Repeat the same procedure for all the objects of interest.

Step 8. Repeat the same procedure for all the frames in the sequence.

Step 9: Stop.

Stop 10: End.

\section{EXPERIMENTAL ANALYSIS AND RESULTS DISCUSSION}

Experiments are carried out on the well known data sets and also captured by our sensor. The algorithm is directly applied to sequences without any preprocessing. This itself is considered as the motion deblurr algorithm. The proposed algorithm is tested on the four image sequences which undergoes various critical issues in the sequence. The data sets are summarized in the Table 1.

Table 1. Input sequence details

\begin{tabular}{|c|c|}
\hline Sequence name & Number of frames considered \\
\hline $\begin{array}{c}\text { Indoor Corridor } \\
\text { sequence }\end{array}$ & 300 \\
\hline $\begin{array}{c}\text { Outdoor animal } \\
\text { moving sequence }\end{array}$ & 180 \\
\hline $\begin{array}{c}\text { Indoor wall clock } \\
\text { pendulum sequence. }\end{array}$ & 150 \\
\hline
\end{tabular}

The table 1 summarizes the input sequence image frames considered for experimental demonstration of proposed method. The indoor corridor sequence consists of single and multiples articulated objects with pose changes moving in the corridor. The second candle sequence. Our third sequence is complex back ground, outdoor animal sequence. Finally, we have considered indoor wall clock pendulum moves continuously. It has high shinning pendulum, which needed correspondence in every image frames.

\subsection{Case 1: Indoor Corridor Sequence}

Figure 3and Figure 4 demonstrate the correspondence of the corridor sequence, which has single color object correspondence in figure 4 and multiple faces correspondences in the Figure 5.
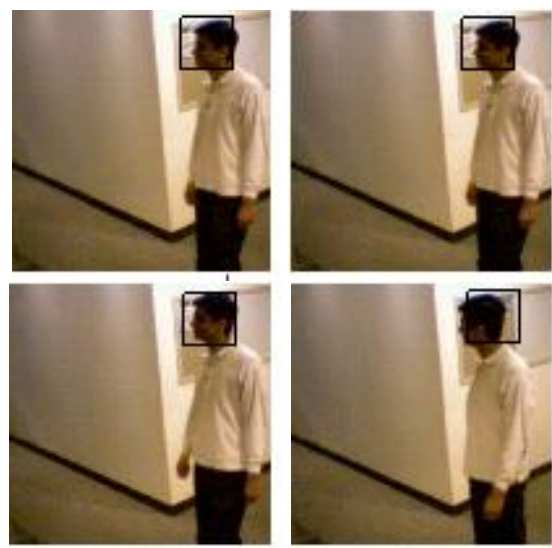

Fig 3. Correspondence of color image sequence

Figure 3 illustrates single object (face) color sequence which has the correspondence with pose changes from right most frame to left most frame. Four frames of the sequence are presented. The frames considered are the consecutive frames of the sequence.
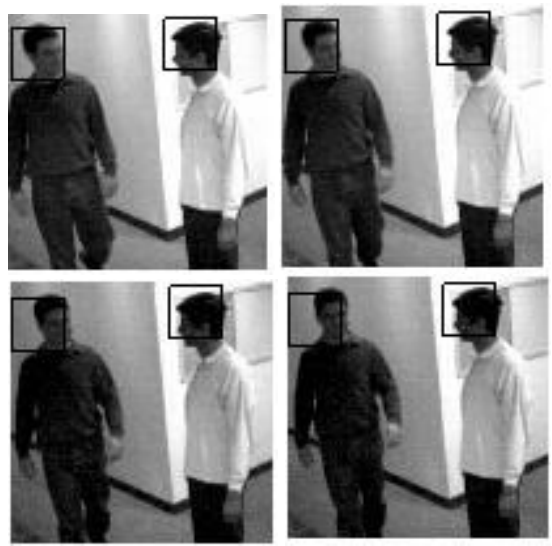

Fig 4. Correspondence of two faces in the sequence

Figure4 illustrates multiobject (faces) correspondence in the five consecutive image frames which undergoes pose changes in both frames. Our algorithm successfully established the correspondence under static sensor, moving object and articulated non-rigid object and it also demonstrates for both single and multiple objects.

\subsection{Case 2: Indoor Burning Candle Sequence}

The sequence considered to demonstrate our proposed methodology is indoor burning candle sequence. We have processed more than 500 frames in this sequence. Figure 5 illustrates the correspondence of burning candle
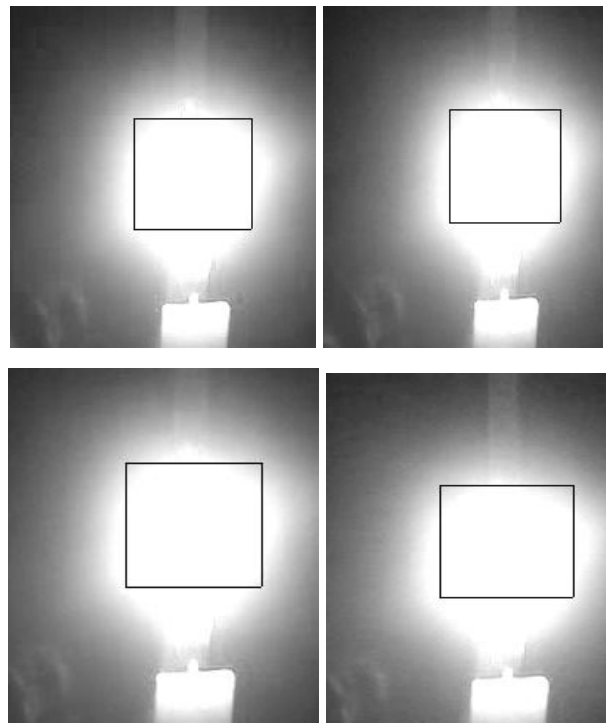

Fig 5. Correspondence of Burning Candle Sequence

Figure 5 consists of 4 frames, which are considered in the middle of the sequence. The burning candle sequence undergoes changes in every frame. Frames presented considered are four frames gap in the sequence. The correspondence established in this sequence is presented inside the rectangular correspondence or control box. Here when ever flame changes in the sequence, the window also changes its position with respect to flame of burning candle. 


\subsection{Case 3: Outdoor Animal Moving Sequence}

The third image sequence considered for correspondence is the moving animal sequence consists of 180 frames for correspondence. It is an outdoor sequence having highly complex back ground involves various different elements with the different intensities. In the Figure 6 we have presented 8 frames of the sequence randomly. The first frame is the reference frame, which does not contain any correspondence window.

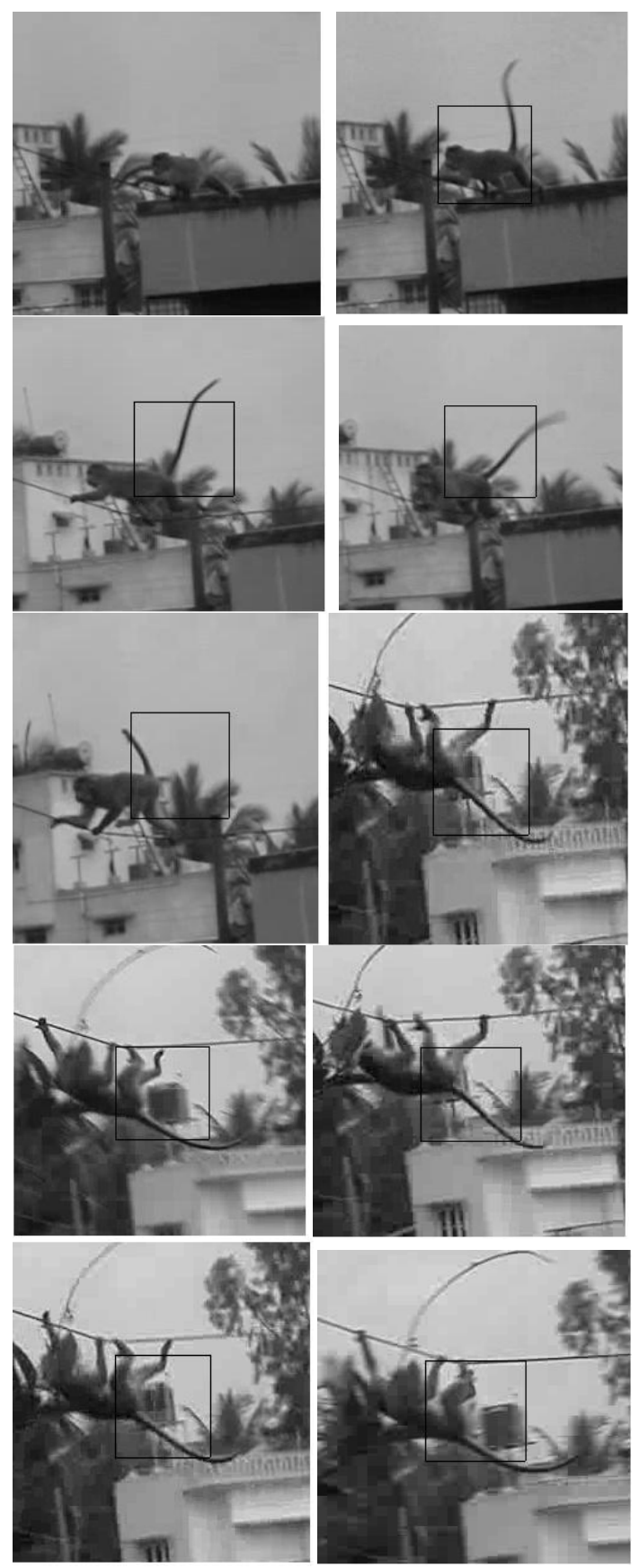

Fig 6. Correspondence of Animal moving sequence in the complex background

Figure 6 illustrates our proposed algorithm's correspondence. The correspondence of monkey is presented from starting position to the various positions are presented. The shape of the monkey itself changes in every frame. The correspondence of monkey is presented inside the square window in all the nine frames from top right corner to bottom left corner. This demonstrates the efficiency of our edge based system working under moving object, moving sensor and heterogeneous dynamic complex background.

\subsection{Case 4: Indoor Wall clock Pendulum Sequence}

$\mathrm{He}$ fourth and final sequence considered for correspondence is the wall clock pendulum. The sequence consists of only one object moving in every second from left to right continuously. We have considered 150 frames of sequence for correspondence. The correspondence object is highly shinning and the surface different intensities in different frames.
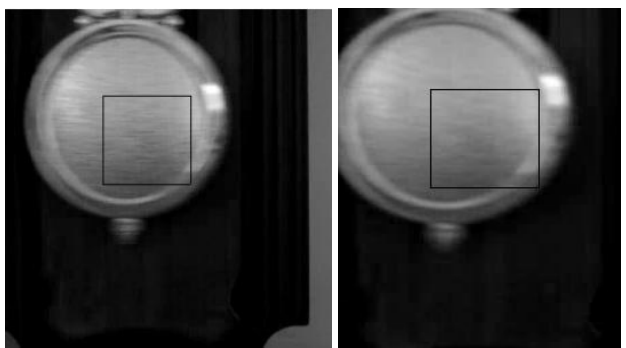

Fig 7. Correspondence for wall clock Pendulum

Figure 7 illustrates the correspondence of pendulum in the four frames. The square window has the correspondence in the frames. This is one of the best illustrations showing efficacy of our edge based feature correspondence algorithm. This works, under high satisfaction for a correspondence problem, related to indoor, static sensor and moving object.

The summary of the qualitative analysis of our method is presented in Table 2.

Table 2. Summary of the qualitative analysis of the proposed method

\begin{tabular}{|c|c|l|}
\hline Techniques & $\begin{array}{c}\text { Features for } \\
\text { considered } \\
\text { Correspondence }\end{array}$ & $\begin{array}{l}\text { Time } \\
\text { complexity } \\
\text { involved }\end{array}$ \\
\hline Proposed method & $\begin{array}{c}\text { Mean of the Canny } \\
\text { edge of Region of } \\
\text { interest }\end{array}$ & $\begin{array}{c}\text { Evaluate edge } \\
\text { and edge's } \\
\text { mean }\end{array}$ \\
\hline $\begin{array}{c}\text { Canny Eigen based } \\
\text { method }\end{array}$ & $\begin{array}{c}\text { Canny edge and its } \\
\text { eigen values }\end{array}$ & $\begin{array}{c}\text { Evaluate } \\
\text { edges and the } \\
\text { polynomial } \\
\text { evaluation of } \\
\text { the edges }\end{array}$ \\
\hline
\end{tabular}

The canny eigen based method has the complexity $\mathrm{O}(\mathrm{nn})$ if $n * n$ is the size of the region of interest. Our proposed method has $\mathrm{O}(\mathrm{n})$ the time taken to find the statistical features. It is a simple addition.

The Table 2 descries the qualitative analysis of our algorithm, which summaries the comparison of our proposed methodology with the canny's eigen values. Our method takes less time to evaluate the features. It does not contain any polynomial evaluations.

Figure 8 represents the comparison of our proposed method with canny eigen based methods. 


\section{Execution time chart}

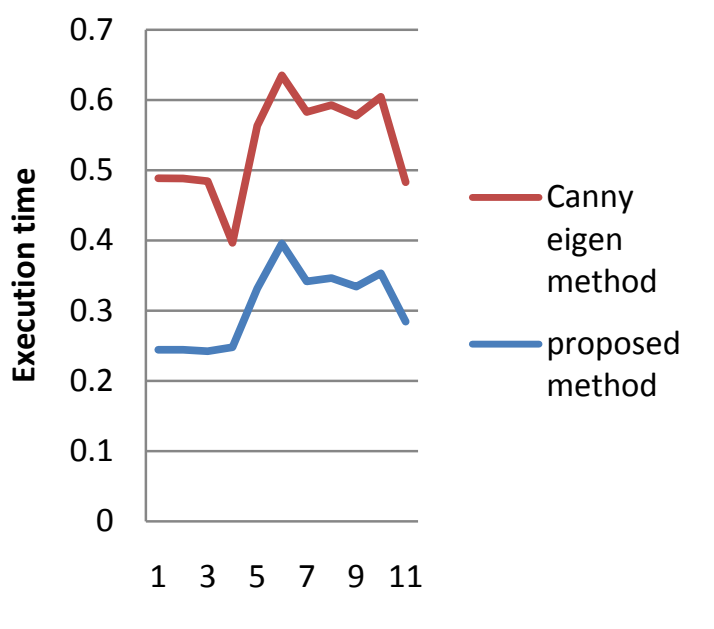

Fig 8. Comparison of proposed approach with canny eigen method

The graph in Figure 9 presents the superiority of our algorithm, which is computationally efficient and inexpensive compared to the canny's eigen based method. Our method does not involve any polynomial evaluation during feature extraction stage. From the graph we conclude that our method is superior. Finding the eigen values for canny's edge is the nth degree polynomial takes more computation time compared out simple statistical mean.

\section{CONCLUSION}

Restoration technology and correspondence tracking of motion are of the important technical areas in image processing. Canny mean restoration and correspondence is one of the novel hybrid approach both for restoration of correspondence and motion tracking of dynamic image sequence analysis. Feature based canny mean features are used to represent the region of interest in the image. The same features are identified in the search image. The correspondence is possible from most similar matching region. It is simple feature representation method. The proposed method is novel method for dynamic image correspondence. The time efficiency of the method is higher than the canny eigen values based registration. The effectiveness of our algorithms is demonstrated on the real world scenes of indoor and outdoor dynamic video sequences. The detailed experimental results are presented for object to image correspondence. The four case studies are presented to demonstrate the computational efficiency of our algorithm. Our algorithm is capable of handling indoor and outdoor scene captured by static sensor dynamic object, dynamic object dynamic sensor with dynamic heterogeneous complex background. Further our algorithm establishes correspondence for rigid and non- rigid articulated objects in the scene. It also works effectively for and single and multiple objects in the scene.

\section{REFERENCES}

[1] Jong-Ho Lee, Yo-Sung Ho, "High Quality Non Blind Image Deconvolution with Adaptive RegularizationImage", J. Vis. Comm. Image R.22,2011, pp.: 653-663.

[2] D.SrinivasaRao, K.SelvaniDeepthi,K. MoniSushma Deep, "Application of Blind Deconvolution and Application for Image Restoration", International Journal of Engineering Science and Technology (IJEST), Vol. 3 No. 3 March 2011, pp: 1878-1884.

[3] AmanpreetKaur, Hitesh Sharma,"Restoration of MRI Images with various types of techniques and Compared with Wavelet Transform",CPMR-IJT, Vol. 2, No. 1, June 2012, pp: 6-12.

[4] BI Xiao-jun, WANG Ting, "Adaptive Blind Image Restoration Algorithm of Degraded Image", IEEECongress on Image and Signal Processing CISP '08, Vol. 3, 2008, pp: 536-540.

[5] Mr. Salem Saleh Al-amri , Dr. N.V. Kalyankar, "A Comparative Study for Deblurred Average Blurred Images",(IJCSE) International Journal on Computer Science and Engineering, Vol. 02, No. 03, 2010, pp: 731-733

[6] Yuanxiang $\mathrm{Li}$, $\mathrm{Na} \mathrm{Li}$,"Image Restoration using Improved Particle Swarm Optimization”,International Conference on Network Computing and Information Security, 2011, pp:394-397.

[7] Feng-qing Qin, Jun Min, Hong-rongGuo, "A blind Restoration Method based on PSF Estimation", IEEEWRI World Congress on Software Engineering WCSE '09, Vol. 2, 2009, pp: 174-176.

[8] Ming Yan, "Restoration of images corrupted by Impulse Noise Using Blind Inpaintingand 10 norm", Preprint, November 7,2011, pp: 1-14.

[9] S. DerinBabacan, Rafael Molina,Aggelos K.Katsaggelos, "Sparse Bayesian Image Restoration", $17^{\text {th }}$ IEEE International Conference on Image Processing (ICIP),Sep. 26-29, 2010, pp:3577-3580.

[10] Hanyu Hong , Liangcheng Li, Luxin Yan, TianxuZhang,"Unified Restoration Method for Different Degraded Images", IEEE International Conference on Optoelectronics and Image Processing (ICOIP),Vol. 2, 2010, pp: 714-717.

[11] Archee NAZ, AnjanTaludar, Kanarpa Kumar Sarma, "Digital Image Restoration using Discrete Wavelet Transform Based approach",IRNet Transactions on Electrical and Electronics Engineering (ITEEE), Vol-1, Iss-2, 2012, pp:53-57.

[12] Liu Yang-Yang, Jin Wei-qi, "Super-Resolution Image Restoration based on Orthogonal Discrete Wavelet Transform", Proc. of SPIE, Vol. 5637,2005, pp: 203-211.

[13] A. Prochazka, J. Ptacek, I. Sindelarova, "Wavelet Transform in Signal and Image Restoration", In Proceedings of Conference CONTROL, 2004, pp:1-5.

[14] Sun qi, Hongzhi Wang, Lu Wei, "An Iterative Blind Deconvolution Image Restoration algorithm based on Adaptive Selection of Regularization Parameters", 
$3^{\text {rd }}$ IEEE International Symposium on Intelligent Information Technology Application,Vol. 1, IITA,2009,pp:112-115.

[15] Ashwini M. Deshpande, SupravaPatnaik, "Comparative study and Qualitative-Quantitative Investigations of Several Motion Deblurring algorithm”, $2^{\text {nd }}$ International Conference and workshop on Emerging Trends in Technology (ICWET), No. 2, 2011, pp: 27-34.

[16] Stuart W. Perry, Ling Guan, "Perception based Adaptive Image restoration", IEEE Acoustics, Speech and Signal Processing, 1998. Proceedings of the 1998 IEEE International Conference, Vol.5, 1998,pp:2893296.

[17] Yong Ge, Qiuming Cheng, "Boundary Effect Reduction in Image Filtering", ICGST International Journal on Graphics, Vision and Image Processing GVIP journal, Vol. 7, Issue 2, Aug 2007, pp: 17-25.

[18] RyuNagayasu, Naoto Hosoda, Nari Tanabe, Hideaki Matsue, Toshihiro Furukawa, "Restoration method for Degraded Images using Two-Dimensional Block Kalman Filter with Colored Driving Source", Digital Signal Processing Workshop and IEEE Signal Processing Education Workshop DSP/SPE,2011, pp:151-156.

[19] Taeg Sang Cho, C. Lawrence Zitnick, Neel joshi,, Sing Bing Kang, Richard Szeliski, William T. Freeman, "Image Restoration by Matching Gradient Distributions", IEEE Transactions on Pattern Analysis and Machine Intelligence, Vol. 34, Issue 4,April 2012, pp. $683-694$.

[20] Sunghyun Cho, Seungyong Lee, "Fast Motion Deblurring", ACM Transactions on Graphics(TOG), Vol.28, No.5, Article 145, Dec. 2009, pp: 145:1$145: 8$

[21] Videre Design-Small Vision System",http://www.videre design.com/vision/svs.htm,2 009.

[22] S.Birchfield and C.Tomasi ,"A Pixel Dissimilarity Measure that is Insensitive image Sampling", IEEE Trans.Pattern Analysis and Machine Intelligence,vol.20, no.4, pp 401-406,Apr.1998.

[23] B.Lofy,"High accuracy Registration and Targeting", Proceedings of the $29^{\text {th }}$ applied imagery pattern recognition workshop, oct. 16-18,2000,pp 235-241.

[24] Serge Beucher,"Watershed, Hierarchical Segmentation And Waterfall Algorithm".
[25] Hidetomo Sakaino and Xiqun Lu,'Dynamic Edge Detection And Mutiple Frame Based Derivative Tensor", pp 2161-2163, ICIP 2009.

[26] L.Brown. A survey of image registration techniques. In ACM Computing Surveys, volume 24, pages 325375, new York, 1992, ACM Press.

[27] Zullfiqar Hasan khan and Irene Yu-Hua Gu, "Joint Feature Correspondence and Appearance Similarity for Robust Object Tracking" IEEE Transac. On information forensic and security, 5(3)September 2010.

[28] Zhengyou Zhang Olivier Faugeras," 3D Dynamic scene Analysis- A stereo Based Approach," SprengerVerlag.

[29] Juyang Weng, Thomas S Huang, Narendra Abuja," Motion and structure from image Sequence," Springler-Verlag.

[30] Edited by S.Levioldi," Multi Computer Vision,' Academic press 1988

[31] Ting Junfan," Describing a Recognizing 3-D objects using surface properties."

[32] Hans-Hermann Bock, Admin Diday (Eds)," Analysis of Symbolic Data," Springer.

[33] Earl Goes, Richard Johnsonbaugh, Steve Jost," Pattern recognition and image Analysis."

[34] Laurent Mallet. "Structural Methods in Pattern Recognition," North Oxford Academic.

[35] Jean-Claude Simon, "Patterns and Operators the foundation of Data Representation," North oxford Academic.

[36] Richard O Duda, PeterE. Hart, David D.Stork, "Pattern Classification" Second Edition, Awiley Inter Science Publication.

[37] Menehem Friendman, Abraham, Kandel," Introduction to Pattern Recognition,"World Scientific.

[38] Robert J.schalkoff, "Patter recognition, Statistical, Structural and Neural approaches," Springer.

[39] Dutta and Mazumdar "Digital Image processing and analysis".

[40] J.P.Marquesdasa,” Pattern Recognition, Statistical, Structural and Neural Approaches," Springer.

[41] Milan Sonok, V aclav Hlavac Roger Boyle," Image Processing, Analysis and Machine vision," Second edition, PWS publisher.

[42] Rafael C. Gonzalez, Richard E. Woods, "Digital image processing," Addison Wesley 